\title{
Transmission of Klebsiella strains and plasmids within and between Grey-headed flying fox colonies
}

\section{Authors}

Ben Vezina ${ }^{1 *}$, Louise M. Judd ${ }^{1}$, Fiona K. McDougall2, Wayne S.J . Boardman ${ }^{3}$, Michelle

L. Power ${ }^{2}$, Jane Hawkey ${ }^{1}$, Sylvain Brisse ${ }^{4}$, J onathan M. Monk ${ }^{5}$, Kathryn E. Holtt,6 and Kelly L Wyres ${ }^{1 *}$

1 Department of Infectious Diseases, Central Clinical School, Monash University, Melboume, Vic toria, Australia

2 Department of Biologic al Sc iences, Macquarie University, NSW 2109, Australia

3 School of Animal and Veterinary Sciences, University of Adelaide, SA 5371, Australia

4 Institut Pasteur, Université de Paris, Biodiversity and Epidemiology of Bacterial Pathogens, Paris, France

5 Department of Bioengineening, University of Califomia, San Diego, CA, United States of Americ a

${ }^{6}$ Department of Infection Biology, Lond on School of Hygiene and Tropical Medic ine, London, UK

*Corresponding authors Ben Vezina (benjamin.vezina@monash.edu); Kelly L. Wyres (Kelly.wyres@monash.edu)

\section{Abstract}

The Grey-headed flying fox (Pteropus poliocephalus) is an endemic Australian fruit bat, known to camy pathogens with zoonotic potential. We recently showed these bats harbour the bacterial pathogens Klebsiella pneumoniae and closely related species in the $K$. pneumoniae species complex (KpSC). However, the dynamics of Klebsiella transmission and gene flow within flying fox colonies were not explored and remain poorly understood.

Here we report a high-resolution genomic companison of $39 \mathrm{KpSC}$ isolates from G reyheaded flying foxes. Illumina whole genome sequences $(n=39)$ were assembled de novo and the Kleborate genotyping tool was used to infer sequence types (STs). Oxford Nanopore sequences were generated for 13 isolates (one for each distinct $\mathrm{ST}$ ) in order to generate high-quality completed reference genomes. Read mapping 
and variant calling was used to identify single nucleotide variants (SNVs) within each ST, using the relevant reference genome. In silico genome-scale metabolic models were generated to predict and compare substrate usage to 59 previously published KpSC models for isolates from human and environmental sources, which indicated no distinction on the basis of metabolic capabilities.

High-resolution genome comparisons identified five putative strain transmission clusters (four intra- and one inter- colony, $n=2-15$ isolates each, $\leq 25$ pairwise SNVs). Inter-colony transmission of Klebsiella africana was found between two flying fox populations located within flying distance. The 13 completed genomes harboured 11 plasmids, all of which showed $37-98 \%$ coverage (mean $73 \%$ ) and $\geq 95 \%$ identity to those previously reported from human-associated KpSC. Comparison of plasmids from different flying fox associated KpSC indicated an interspecies horizontal plasmid transmission between $K$. pneumoniae and $K$. africana for a $98 \mathrm{kbp}$ plasmid, pFF1003.

These data indicate that KpSC are able to transmit directly via flying fox populations or indirectly via a common source, and that these isolates can harbour plasmids with similarity to those found in human derived $\mathrm{KPSC}$, indicating gene flow is occuming between isolatesfrom Grey-headed flying fox KpSC and human clinic al isolates.

\section{Introduction}

The Klebsiella pneumoniae spec ies complex (KpSC) [2] is a group of closely-related Klebsiella species which includes Klebsiella pneumoniae, Klebsiella quasipneumoniae subsp. quasipneumoniae, Klebsiella variicola subsp. variicola, Klebsiella quasipneumoniae subsp. similipneumoniae, Klebsiella variicola subsp. tropica, Klebsiella quasivariicola and Klebsiella africana. KpSC are problematic as they function as cosmopolitan opportunistic pathogens [3, 4], responsible for a womying proportion of community and hospital-acquired infections [5]. Prevalence of multi-drug resistance and acquired virulence factors associated with invasive infections is inc reasing over time [6], hence identifying the reservoirs of problematic KpSC lineages/sequence types (STs), and mobile antimicrobial resistance and virulence determinants, is key for targeting interventions to limit the spread of these orga nisms.

Aside from humans, KpSC have also been isolated from a wide variety of environments including soil [7], plants [3, 8] fresh water [7, 9], marine environments and organisms [10], waste dumps [11], animals including cats and dogs [12], migratory and domesticated birds [13, 14], a gricultural animals [15, 16] and Greyheaded flying foxes [1]. Flying foxes, or fruit bats, have long been considered important vectors of zoonotic viruses such as Australian bat lyssavirus [17], Menangle virus [18] and Hendra virus (though not as the primary resenoir) [19]. There is growing evidence that Grey-headed flying foxes also harbour human pathogenic bacteria such as antibiotic resistant and pathogenic Escherichia coli lineages [20]. Carbapenem resistant $K$. pneumoniae have also previously been isolated from insectivorous bat guano in Algeria [21], though at very low frequency (two positive out of 110 samples). 


\section{FGURE 1}

Our previous surveillance study [1] identified KpSC from three of four wild flying fox colonies (up to $36 \%$ prevalence per colony) and a captive rehabilitation colony (80\% prevalence) in Australia (Figure 1). Notably, this was the first non-human sighting of $K$. africana, a species which has been reported in just two previous studies globally [6, 22]. Our initial genomic a nalyses of these KpSC strains from flying foxes indicated low ST diversity with few STs previously reported among human clinical isolates, as well as low rates of antimic robial resistance (1.1\%) and virulence factors, suggesting that these Grey-headed flying fox colonies are not a high-risk environmental reservoir for problematic KpSC isolates. However, this a nalysis did not explore detailed strain relationships, nor the broader genetic content of these KpSC.

Here, we use long-read DNA sequencing to complete the genomes of $13 \mathrm{KpSC}$ from flying foxes and leverage these data alongside the previously reported draft genomes to perform the first high-resolution analysis of KpSC strain and plasmid transmission dynamics within/between Grey-headed flying fox colonies. To better understand the potential for strain and plasmid transfer into the human population, we compare completed plasmid sequences from flying fox isolates to those identified among human-associated KpSC, and utilise the latest genome-scale metabolic modelling approaches to compare the metabolic capabilities of flying fox derived isolates to KpSC isolatesfrom other sources.

\section{Results}

\section{High-resolution SNV analysis identified multiple putative strain transmissions}

Among the 13 distinct KpSC STs previously reported from our flying fox isolate collection, five were associated with $>1$ isolate each (Figure 2), four of which comprised pairs or groups of isolates that differed by $\leq 25$ SNVs, indic a tive of recent stra in transmissions [31-33]. Mapping data can be found in Table $\mathbf{S 2}$, while pairwise SNVscan be found in Table $\mathbf{5 3}$, ranging from 0-715.

There were three occurrences of putative intra-colony transmission at the Camellia gardens colony, including $K$. pneumoniae ST1017 ( $n=2$ isolates, three painwise SNVs), K. africana ST4938 ( $n=3$ isolates, zero pairwise SNVs) and K. pneumoniae $\operatorname{ST5033~(~} n=4$ isolates, $\leq 2$ painwise SNVs), and one occurrence at the captive Mylor colony of $K$. pneumoniae 515035 ( $n=15$ of 16 isolates recovered from this colony, $\leq 3$ painwise SNVs) (Figure 2). A single putative $K$. africana ST4938 inter-colony transmission event was observed between the Camellia Gardens and Blackalls Park colonies $(n=2$ isolates, 21 painwise SNVs). Notably, this inter-colony cluster was separated from the Camellia Gardens ST4938 intra-colony cluster and two unclustered isolates by just 36- 
58 pairwise SNVs, which may be indicative of longer-term circulation within the colony and/or acquisition from a common long-tem reservoir (also see ST4938 phylogeny below).

Two K. pneumoniae ST4919 isolates, one from the Adelaide colony and the other from the Camellia Gardens colony (approximately 1,150 kilometres apart), were separated by 715 SNVs.

\section{FGURE 2}

\section{Plasmid diversity and transmission between species}

A total of 11 plasmids were identified across the 13 completed genomes. Two isolates ( $K$. africana ST4938, stra in FF1003 and $K$. pneumoniae ST5033, strain FF979) harboured two plasmids each, seven isolates harboured a single plasmid each, while four isolates contained none (Table $\mathbf{5 2}$ ). The plasmids ranged from approximately 5 kilobases (kb) to $175 \mathrm{~kb}$ in length (Figure 3, Table S4). At least 7 of the 11 plasmids were predicted to be either self-conjugative or mobilizable and only one contained any antimic robial resistance genes ( $K$. pneumoniae ST1017, stra in FF1009_1, camying qnrS1 and a variant of dfrA14) and none contained any of the well characterised KpSC virulence genes [2]. Nonetheless, all plasmids demonstrated high identity ( $\geq 95 \%$ ) with generally high coverage (37-98\%; mean $73 \%$ ) to at least one plasmid from human-derived KpSC isolates (Figure 3).

A reference-based mapping approach was used to detemine the presence or absence of each of our completed plasmids among host isolates of the same ST, which revealed a high level of conservation ( $\geq 78 \%$ mapping coverage in all but three instances, comprised of three out of $7 \mathrm{~K}$. africana ST4938 isolates missing the pFF1003_2 plasmid) (Table S2). At most, plasmids differed amongst themselves by eight pairwise SNVs (Table S3). Notably, these included the pFF1009_1 AMR plasmid identified in both $K$. pneumoniae ST1017 isolates, and the $\sim 5.5 \mathrm{~kb}$ pFF1043_1 plasmid identified among the $K$. pneumoniae 5 T4919 isolates collected from two independent colonies (Adelaide and Camelia Gardens). These contained zero and one pairwise SNVs to the completed pFF1009_1 and pFF1043_1, respectively.

Read mapping analyses indicated that a pFF1003_1-like conjugative plasmid was present in nine of the total 39 isolates (23\%) (including those from both the Camellia Gardens and Blackalls Park colonies, maximum 8 pairwise SNVs). These included representatives of $K$. africana ST4938, as well as K. pneumoniae ST5037 and $S T 1412$ (Figure 3, Figure 4). Comparison of the completed sequences for pFF1003_1 (mapping reference from FF1003, a $K$. africana ST4938 isolate) and pFF1010_1 (from FF1010, a K. pneumoniae ST1412) showed 99\% BLASTn coverage and 99\% identity, indicative of recent plasmid transmission, though SNV distances were not calculated. However, a comparison between the pFF1003_1 reference and the completed sequence for pFF1023_1 (from FF1023, a K. pneumoniae ST5037) showed only $77 \%$ BLASTn coverage and $99 \%$ identity. Approximately 9 kb of pFF1023_1 was deleted compared to pFF1003_1 (at least six independent deletion events), 
including part of the conjugation locus. As such, this plasmid was predicted to be non-conjugative. The pFF1023_1 nan locus (consisting of nanA, nanT, nanE2, nanK, yhcH, a putative $\mathrm{N}$-acetylneuraminic acid porin (Genbank accession: HAT1683918.1), nanC and nanM, flanked by IS3 family transposases), was also inverted compared to the pFF1003_1 reference plasmid (Figure 4C).

pFF1003_1 (97 kb) showed 64\% BLASTn coverage and 99\% identity to the publicly available $141 \mathrm{~kb}$ human-derived KpSC plasmid (pINF116_1). This plasmid was identified from $K$. pneumoniae ST36 strain INF116, isolated from an Australian human urine sample in 2013 (Genbank accession: CP031793.1). Compared with pINF116, pFF1003_1 lacked many of the amino acid transport genes such as hisQ, hisJ and $g \ln Q$, but contained the additional tyrosine recombinase xerC, tRNA(fMet)-specific endonuclease $v a p C$ and the putatively intact sialic acid degradation nan locus (Figure 4C). 
AGURE 3

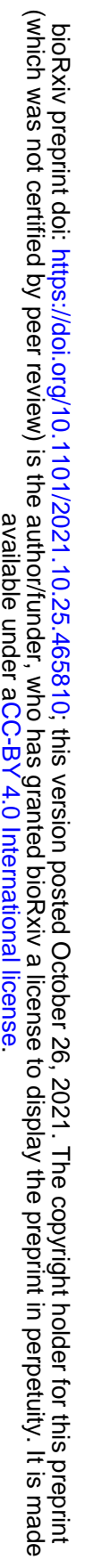


RGURE 4

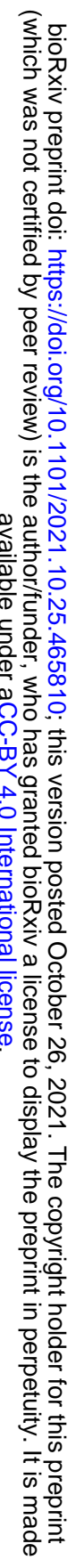




\section{KpSC from flying foxes were not distinguished by metabolic capabilities}

We simulated growth phenotypes in silico and overlaid these on the core genome phylogeny of $72 \mathrm{KpSC}$ genomes to identify any potential genetic or metabolic commonalities of KpSC isolates from Grey-headed flying foxes compared to those from other sources. All KpSC isolates were predicted to utilise a core set of substrates accounting for $49.6 \%$ of carbon, $40 \%$ nitrogen, $72.9 \%$ of phosphorus and $40 \%$ of sulphur substrates tested (Table S5, Table S6). Percentage of total nutrient sources tested which displayed variable usage (non-core) across the KpSC isolates were much lower, consisting of $10.7 \%$ carbon, 3.2\% nitrogen, $1.7 \%$ phosphorus and $0 \%$ sulphur sources.

We generated a core gene phylogeny and overlaid the variable substrate usage profiles to examine the relationship between lineage and metabolic substrate usage (Figure 5, Figure S1). Isolates from flying foxes were distributed throughout the phylogeny and predicted growth phenotypes appeared to cluster by species, then more granularly by ST (Figure 5), rather than isolate source. Hiera rchic al clustering of isolates based on predicted growth phenotypes was also consistent with this observation (Figure $\mathbf{5 2}$ ), with isolates of the same species generally clustering together. One isolate, K. pneumoniae DHQP1002001 appeared as an outgroup to the other $K$. pneumoniae isolates in the phylogeny. This appeared to be a genetic species hybrid of mostly $K$. pneumoniae with roughly $1 \mathrm{Mb}$ from $\mathrm{a} K$. quasipneumoniae subsp. quasipneumoniae strain (Figure S3). Notably, unlike the rest of the $K$. pneumoniae isolates, this species hybrid was predicted to be unable to utilise ethanolamine and D-glucarate as sole sources of carbon. While $K$. quasipneumoniae subsp. quasipneumoniae isolates were universally predicted to be unable to utilise the ethanolamine, they were all predicted to utilise tricarballylate and D-glucarate. It is unclear whether this pattem of substrate usage loss exists for Klebsiella hybrid speciesin general or waslimited due to the data used. 
bioRxiv preprint doi: https://doi.org/10.1101/2021.10.25.465810; this version posted October 26, 2021. The copyright holder for this preprint (which was not certified by peer review) is the author/funder, who has granted bioRxiv a license to display the preprint in perpetuity. It is made available under aCC-BY 4.0 International license.

\section{FGURE 5}




\section{Disc ussion}

Our high-resolution genomic comparison of KpSC from Grey-headed flying fox colonies a cross Australia indic ates strong evidence for stra in transmission both within and between colonies (Figure 2). Five putative strain transmission clusters were identified, four of which involved the wild Camellia Gardens colony in the state of New South Wales. This included three clusters contained within this colony and one transmission cluster between this colony and the wild colony at Blackalls Park. The latter was associated with K. africana ST4938 for which isolates FF1017 (Camelia Gardens) and FF930 (Blackalls Park), were separated by just 21 chromosomal SNVs (Figure 2) and shared a pFF1003_1-like ( $-98 \mathrm{~kb})$ plasmid with 0 pairwise SNVs, indicating they descended from a very recent common ancestor (although a second plasmid present in FF1017 was absent from FF930). Notably, these colonies a re situated $123 \mathrm{~km}$ apart (Figure $\mathbf{1}$ ) and were both sampled within a 23-day period. As Grey-headed flying foxes have been shown to travel large distances of $\sim 50 \mathrm{~km}$ [67] and up to $>1,000 \mathrm{~km}$ in some cases [68], it is possible for individual flying foxes to move between the Blackalls Park and Camellia Gardens colonies causing direct inter-colony transmission. Altematively, transmission may have occurred ind irectly via a common source (food/water etc). Additionally, our analyses indic ated that the two ST4938 clusters, as well as two additional unclustered ST4938 isolates from Camellia Gardens differed by no more than 58 pairwise SNVs (Figure 2, Table $\mathbf{5 3}$ ). These values are much lower than would be expected for randomly selected KpSC of the same ST $[32,69]$ and similar to those observed for isolates representing longerterm transmission within hospital networks [32, 69]. Hence, these findings are consistent with longer-term transmission in the flying fox population or acquisition from the same long-term reservoir.

The fifth putative strain transmission cluster was identified at the captive Mylor colony located within a rehabilitation centre, which at the time of sampling was a closed population of 30 - 40 adult Grey-headed flying foxes from the local wild Adelaide Botanic Park colony that had been affected by a heat stress event. These unhealthy individuals may have suffered increased susceptibility to opportunistic colonisation and dissemination of KpSC isolates e.g. from other wild life or domestic animals that were housed in close proximity, which we speculate facilitated the broad scale dissemination of $K$. pneumoniae $5 T 5035$ in the population (isolated from 15 of 20 sampled individuals [1]).

There is currently very limited data on the dynamics of bacterial transmission among flying foxes and other bat species; however, our results align with a previous study of viral dynamics [70], which found transmission of Nipah virus in Indian flying foxes within Bangladesh occurred within and between colonies. This study also noted the cycles of Nipah virus prevalence could be influenced by colony demographics, such as individual bat turnover which reduced herd immunity [70]. Grey-headed flying fox colonies in New South Wales have an average daily individual tumover of $17.5 \%$ as they travel between roosts via a node-based network [71], indicating high influx and efflux of individuals. This behavioural activity may make Grey-headed flying fox colonies susceptible to between-colony transmission of mic robes such as 
KpSC, however further sampling is required to quantify these transmission events and detemine if they represent transient or ongoing phenomena.

In addition to strain transmissions, we identified putative transmission of a $98 \mathrm{~kb}$ plasmid, passing the species bamier between $K$. africana ST4938 and K. pneumoniae ST1412 and ST5037 (Figure 3, Figure 4). The plasmid variants harboured by FF1003 (K. africana ST4938) and FF1010 ( $K$. pneumoniae ST1412) were highly similar (4 pairwise SNVs, $100 \%$ coverage and $99 \%$ nucleotide identity), supporting a recent transmission event. In contrast, the plasmid variant harboured by FF1023 ( $K$. pneumoniae ST5037) showed at least six deletions as well as a structural rearrangement, indicating a plasmid transmission likely occurred at some point in the more distant evolutionary history of these strains (either directly or indirectly via intermediary hosts). In that regard, it is possible that the pFF1003-like plasmid may have been circulating within the Camelia Gardens colony for some time and/or has been introduced from a common reservoir multiple times. Aside from the presence of several selfish genetic elements including a higAB toxin-antitoxin system, intact conjugative tra locus (except in the case of pFF1023_1) (Figure 4C), this pFF1103-like plasmid contained a putatively intact nan sialic acid locus that was conserved in all variants. The nan locus encodes degradation of $\mathrm{N}$-acetylneuraminic acid, a sialic acid [72]. Utilisation and acquisition of $\mathrm{N}$-acetylneuraminic acid improves colonisation and provides competitive growth advantages to a variety of pathogens [73-75]. It is possible that the presence of this locus provided some sort of selective advantage to promote the transmission and longer-tem maintenance of the pFF1103-like plasmid in the Camelia Gardens population, although further investigations would be required to confirm this hypothesis.

Plasmid pFF1003 also showed high similarity to a plasmid identified from INF116, a $\mathrm{K}$. pneumoniae clinical isolate causing a uninary tract infection in a patient in a Melboume hospital in 2013 [76]. In fact, our search of publicly available nucleotide sequence data indicated that all completed plasmids from flying fox derived KpSC shared high identity ( $\geq 95 \%$ ) with generally high coverage (37-98\%; mean $73 \%$ ) (Table S4) to at least one other plasmid identified from human clinical isolates. Hence, our data provide clear evidence for the flow of genetic material either directly or indirectly between KpSC isolated from flying foxes and human specimens, although we cannot speculate on the directionality or time-frame of transfer. In order for direct transfer to occur these isolate populations would need to coexist, at least transiently, within the same spatial-temporal and ecologic al niche.

While much more work is required to fully understand the distribution and flow of KpSC strains and genetic material between niches (including detailed genomic analysis of large contemporaneous isolate collections), the results of the preliminary metabolic analyses presented here are consistent with nic he overlap (i.e. KpSC from flying foxes did not appear to be metabolically distinct). However, we note that our analysis was constrained to a small sample size and that there are likely many metabolic traits which are uncaptured within the metabolic models used in this study, due to limitations of the current input database. Over time, this approach should become more robust as databases become more complete (https://www.genome.jp/kegg/docs/upd map.html) [77]. There may also be other genetic differences between flying fox and human strains of KpSC such as K/O loci, 
previously disc ussed within the context of flying fox isolates by McDougall et al [1] however due to a paucity of Grey-headed flying fox isolates, we lack the statistical powerto discem this.

\section{Experimental procedures}

\section{Bacterial isolate collection and whole-genome sequenc ing}

Thirty-nine KpSC isolates including thirty $K$. pneumoniae, one $K$. variicola subsp. variicola and eight $K$. africana were previously isolated from four different Greyheaded flying fox colonies [1]; Blackalls Park, sampled on 12/12/2017 and 10/4/2018; Camellia Gardens, 4/1/2018; Adelaide Botanic Park, 11/2/2018, Mylor (captive colony), 12/2/2018; Centennial Park, 9/3/2018. One isolate ( $K$. pneumoniae FF996) was not utilised in this study due to DNA contamination issues identified during highresolution analysis. See Table $\mathbf{S 1}$ for details of all isolates, genotyping results and genome accessions.

Illumina (short-read) whole-genome sequencing data was generated and reported previously [1]. DNA was extracted for long-read sequencing of 13 isolates representing distinct STs (see Table S1), using the GenFind v3 kit (Beckman Coulter), followed by library preparation using the Ligation library kit (SQ K-LSK109, Oxford Nanopore Technologies, ONT) with Native Barcode Expansion (EXP-NBD196, ONT) to allow for multiplexing of libraries. DNA was sequenced on MinION flow cells (R9.4.1, ONT) and basecalled using Guppy version 4.0.14+8d3226e with the dna_r9.4.1_450bps_hac model (ONT).

Illumina-only genome assemblies were generated as reported previously [1]. ONT reads were assembled using Flye version 2.8.1-b1676 [23] (options: --plasmids -g 5250000 --asm-coverage 60) then polished twice with medaka version 1.0.3 [24] using the medaka_consensus module (options: -m r941_min_fast_g303). Finally, assemblies were short-read error corrected using Pilon version 1.23 [25] (two rounds pergenome).

Assemblies were annotated using Prokka version 1.14.6 [26], and assembly statistic s were generated using the statswrapper.sh module from bbmap version 38.81 [27]. Species, multi-locus sequence types, antimicrobial resistance and virulence determinants were identified using Kleborate version v2.0.1 [6]. Suspected hybrid species were analysed in further detail using GenomePainter version 0.0.8 (https://github.com/sc watts/genome painter) [28].

\section{Single nucleotide variant analyses}

Single nucleotide variants (SNVs) were identified using RedDog version 1b.10.4 [29]. Isolates were grouped by ST, and the corresponding Illumina reads mapped to the respective completed chromosome and plasmid reference sequences (Table S1). As a control, the short-read data from the ST reference isolate was also mapped to its completed assembly. Pairwise SNV distances were calculated for each replic on using the snp2dist script at Figshare (https://doi.org/10.6084/m9.figsha re.16609054) [30]. Painwise chromosomal SNV distances $\leq 25$ were considered indicative of recent 
stra in transmission events, based on previous a nalyses of KpSC transmission in clinic al settings [31-33]. A SNV maximum likelihood phylogenetic tree was also constructed from the SNV alignment using RAXML with a GTR substitution model [30].

\section{Plasmid analyses}

Completed plasmid sequences were analysed using the mob_typer command from Mob-suite version 3.0.0 [34]. The closest matching (best query coverage and identity) plasmid sequences from human-derived Klebsiella isolates were identified using NCBI BLASTn search against the public NCBI nucleotide database as of 01/04/21 [35]. A MASH distance tree was generated using mash version 2.1.1 [36], along with $\mathrm{R}$ packages ape version 5.4-1 [37] and phangom version 2.5 .5 [38]. The tree was inferred using the FastME algorithm [39] [30].

Visualisations of painwise plasmid comparisons were generated via EasyFig version 2.2.5 [40] and the BLASTn command, with the following BLAST parameters: Min. length - 1000. Max. e Value - 0.0001, to look at gene content and synteny.

\section{Genome scale metabolic reconstructions and in silico growth predictions}

Metabolic diversity of KpSC from flying foxes ( $n=13$ completed genomes only) was explored, in comparison to KpSC from other host and/or environmental sourc es for which metabolic reconstructions have been published previously ( $n=37$ from [41] and $n=22$ from [42].

Novel metabolic network reconstructions were generated using cobrapy version 0.20 .0 [43] in a conda environment version 4.9.2 [44], running Python 3.6.12 [45]. The KpSC pan metabolic model [41] was used as a reference as previously described $[46,47]$. The specific code used to generate models was adapted from [47] and can be found at Figshare (https://doi.org/10.6084/m9.figshare.16609054) [30]. The same approach was performed to update the $22 \mathrm{~K}$. pneumoniae reconstructions described in [42]. Model annotations were improved using models iYS1720 [48], iM L1515 [49] and iWFL_1372 [50] with a custom script [30]. MEMOTE reports [51] were then generated for each model using the report snapshot option. In silico growth capabilities were simulated in minimal media with each of 268 sole carbon, 154 nitrogen, 25 sulfur and 59 phosphorus sources using the flux balance analysis model.optimize method from cobrapy as described previously [41]. All genome accessions, model identifiers a re shown in Table $\mathbf{S 1}$.

To contextualise the metabolic modelling results with regards to population structure, a core genome phylogeny was inferred from a core gene alignment generated with Panaroo version 1.1.2 [52] (options: --mode relaxed -a core --aligner maff -core_threshold 0.98 -f 0.90 --merge_paralogs). The resulting alignment of 574,339 variant sites represented 3,319 genes shared by $\geq 71 / 72$ isolates. RAxML-NG version 1.1.0 [53] was used to infer a maximum likelihood phylogeny with 1000 bootstrap replicates (options: --all --model GTR), best of five runs. RAXML was also used to generate an unscaled, daylight phylogenetic tree in the contexts of plasmid presence [30]. 


\section{Data visualisation}

Data were visualised using R version 4.0.3 [54] and RStudio version 1.3.1093 [55] with the following software packages: RColorBrewer version 1.1-2 [56], ggplot2 version 3.3.3 [57], reshape2 version 1.1.4 [58], aplot version 0.0.6 [59] and ggnewscale version 0.4.5 [60] [30]. igraph version 1.2.6 [61], ggraph version 2.0.5 [62] and ggforce version 0.3.3 [63] were used for painwise SNV analyses only. ggtree version 2.4.1 [64] was used for phylogenetic tree visualisation only. pheatmap version 1.0.12 [65], grid version 4.0.3 and gridExtra version 2.3 [66] were used for Flux Balance Analysis visualisation.

\section{Data availability}

These sequence data have been submitted to the Genbank databases under BioProject accession number PRJ NA646592. All other data used in this study can be found in supplemental tables and at Figshare (https:// doi.org/ 10.6084/ m9.fig sha re.16609054) [30].

\section{Competing Interests Statement}

The authors declare that they have no competing interests.

\section{Acknowledgements}

This work was supported, in whole or in part, by the Bill \& Melinda Gates Foundation [OPP1175797]. Under the grant conditions of the Foundation, a Creative Commons Attribution 4.0 Generic License has already been assigned to the Author Accepted Manuscript version that might a rise from this submission. This work wa s a lso supported by Australian Research Council Disc overy Project DP200103364.

KLW is supported by the National Health and Medic al Research Council of Australia (Investiga tor Grant APP1176192).

\section{References}

1. McDougall, F.K., et al., Novel stra ins of Klebsiella africana and Klebsiella pneumoniae in Australian Fruit Bats (Pteropus poliocephalus). Resea rch in Microbiology, 2021: p. 103879.

2. Wyres, K.L., M.M.C. Lam, and K.E. Holt, Population genomic s of Klebsiella pneumoniae. Nature Reviews Mic robiology, 2020. 18(6): p. 344-359.

3. Knittel, M.D., et al., Colonization of the Botanic al Environment by Klebsiella Isolates of Pathogenic Origin. Applied and Environmental Microbiology, 1977. 34(5): p. 557-563. 
4. Navon-Venezia, S., K. Kondratyeva, and A. Carattoli, Klebsiella pneumoniae: a major worldwide source and shuttle for antibiotic resistance. FEMS Mic robiology Reviews, 2017. 41(3): p. 252-275.

5. Pendleton, J.N., S.P. Goman, and B.F. Gilmore, Clinical relevance of the ESKAPE pathogens. Expert Review of Anti-infective Therapy, 2013. 11(3): p. 297-308.

6. Lam, M.M.C., et al., A genomic surveillance framework and genotyping tool for Klebsiella pneumoniae and its related speciescomplex. Nature Communic ations, 2021. 12(1): p. 4188.

7. Melo-Nasc imento, A.O.d.S., et al., Functional cha rac terization of ligninolytic Klebsiella spp. strains associated with soil and freshwater. Archives of Microbiology, 2018. 200(8): p. 1267-1278.

8. Rosenblueth, M., et al., Klebsiella variicola, A Novel Species with Clinic al and Plant-Associated Isolates. Systematic and Applied Microbiology, 2004. 27(1): p. 27-35.

9. Hladicz, A., C. Kittinger, and G. Zarfel, Tigecyc line Resistant Klebsiella pneumoniae Isolated from Austrian River Water. Intemational J oumal of Environmental Research and Public Health, 2017. 14(10).

10. Håkonsholm, F., et al., Antibiotic Sensitivity Sc reening of Klebsiella spp. and Raoultella spp. Isolated from Marine Bivalve Mollusc s Reveal Presence of CTXM-Producing K. pneumoniae. Mic roorga nisms, 2020. 8(12): p. 1909.

11. Awasthi, S., et al., Biodegradation of themally treated high-density polyethylene (HDPE) by Klebsiella pneumoniae CH001. 3 Biotech, 2017. 7(5): p. 332.

12. Marques, C., et al., Klebsiella pneumoniae causing urinary tract infections in companion a nima Is and humans: population structure, a ntimic robial resistance and virulence genes. J oumal of Antimic robial Chemotherapy, 2019. 74(3): p. 594-602.

13. Liao, X., et al., High colonization rate of a novel carba penem-resistant Klebsiella linea ge a mong migra tory bird s at Q inghai Lake, China. J oumal of Antimic robial Chemothera py, 2019. 74(10): p. 2895-2903.

14. Ahmed, H.A., et al., Pet birds as potential reservoirs of virulent and a ntibiotic resistant zoonotic bacteria. Comparative Immunology, Microbiology and Infectio us Disea ses, 2021. 75: p. 101606.

15. Ha mza, E., S.M. Dorgham, and D.A. Hamza, Carba penemase-producing Klebsiella pneumoniae in broiler poultry farming in Egypt. J oumal of G lobal Antimic robial Resistance, 2016. 7: p. 8-10.

16. Massé, J., S. Dufour, and M. Arc ha mbault, Cha racterization of Klebsiella isolates obtained from clinical mastitis cases in dairy cattle. J oumal of Dairy Science, 2020. 103(4): p. 3392-3400.

17. Fraser, G.C., et al., Enc ephalitis Caused by a Lyssavirus in Fruit Bats in Australia. Emerging Infectious Disease joumal, 1996. 2(4): p. 327.

18. Philbey, A.W., et al., Infection with Menangle virus in flying foxes (Pteropus spp.) in Australia. Australian Veterinary J oumal, 2008. 86(11): p. 449-454.

19. Halpin, K., et al., Isolation of Hendra virus from pteropid bats: a natural reservoir of Hendra virus. J oumal of General Virology, 2000. 81(8): p. 19271932.

20. McDougall, F.K., W.S.J . Boardman, and M.L. Power, Characterization of betala ctam-resistant Escherichia coli from Australian fruit bats indic a tes anthropogenic origins. Mic robial Genomic s, 2021. 7(5). 
21. Gharout-Sait, A., et al., Occurrence of Carbapenema se-Producing Klebsiella pneumoniae in Bat Guano. Microbial Drug Resistance, 2019. 25(7): p. 10571062.

22. Rodrigues, C., et al., Desc niption of Klebsiella africanensis sp. nov., Klebsiella variicola subsp. tropicalensis subsp. nov. and Klebsiella variicola subsp. variicola subsp. nov. Research in Mic robiology, 2019. 170(3): p. 165-170.

23. Kolmogorov, M., et al., Assembly of long, error-prone reads using repeat graphs. Nature Biotechnology, 2019. 37(5): p. 540-546.

24. nanoporetech. Medaka. 2020 Accessed: 10/12/2020; Version 1.0.3:[Availa ble from: https://github.com/nanoporetech/medaka.

25. Walker, B.J ., et al., Pilon: An Integrated Tool for Comprehensive Microbial Variant Detection and Genome Assembly Improvement. PLOS ONE, 2014. 9(11): p. e112963.

26. Seemann, T., Prokka: rapid prokaryotic genome annotation. Bio informatics, 2014. 30(14): p. 2068-2069.

27. Bushnell, B. BBMap: A Fast, Accurate, Splic e-Awa re Aligner. 2014. Available from: https:// sourc eforge.net/projects/bbmap/.

28. Watts, S. GenomePainter. 2018, v 0.0.8. Available from: https://github.com/sc watts/genome painter.

29. Edwards, D., B. Pope, and K. Holt. RedDog 2016, v 1b.10.4. Available from: https://github.com/katholt/RedDog.

30. Vezina, B., et al. Klebsiella genome-scale metabolic models isolated from Grey-headed flying foxes. 2021 Figshare; https:// d oi.org/ 10.6084/m9.figsha re.16609054

31. Gomie, C.L., et al., Gastrointestinal Ca mia ge Is a Major Reservoir of Klebsiella pneumoniae Infection in Intensive Care Patients. Clinical Infec tious Disea ses, 2017. 65(2): p. 208-215.

32. David, S., et al., Epidemic of carbapenem-resistant Klebsiella pneumoniae in Europe is driven by nosoc omial spread. Nature Microbiology, 2019. 4(11): $p$. 1919-1929.

33. Shemy, N.L., et al., Pilot study of a combined genomic and epidemiologic surveillance program for hospital-acquired multidrug-resistant pathogens across multiple hospital networks in Australia. Infection Control \& Hospital Epidemiology, 2021. 42(5): p. 573-581.

34. Robertson, J. and J.H.E. Na sh, MOB-suite: softwa re to ols for clustering, reconstruction and typing of plasmids from draft a ssemblies. Microbial Genomics, 2018. 4(8).

35. J ohnson, M., et al., NCBI BLAST: a better web interface. Nucleic Acids Research, 2008. 36(suppl_2): p. W5-W9.

36. Ondov, B.D., et al., Mash: fast genome and metagenome distance estimation using MinHash. Genome Biology, 2016. 17(1): p. 132.

37. Paradis, E., J . Claude, and K. Strimmer, APE: Analyses of Phylogenetic s and Evolution in R language. Bioinformatics, 2004. 20(2): p. 289-290.

38. Schliep, K.P., phangom: phylogenetic a nalysis in R. Bioinformatics, 2011. 27(4): p. 592-593.

39. Lefort, V., R. Desper, and O. Gascuel, FastME 2.0: A Comprehensive, Accurate, and Fast Distance-Based Phylogeny Inference Program. Molecular Biology and Evolution, 2015. 32(10): p. 2798-2800.

40. Sullivan, M.J., N.K. Petty, and S.A. Beatson, Easyfig: a genome comparison visualizer. Bioinformatic s, 2011. 27(7): p. 1009-1010. 
41. Hawkey, J., et al., A curated collection of Klebsiella metabolic models reveals variable substrate usage and gene essentiality, bioRxiv, 2021: $p$. 2021.10.10.463858.

42. Norsigian, C.J., et al., Comparative Genome-Sc ale Metabolic Modeling of Metallo-Beta-Lactamase-Producing Multidrug-Resistant Klebsiella pneumoniae Clinic al Isolates. Frontiers in Cellular and Infection Microbiology, 2019. 9(161).

43. Ebrahim, A., et al., COBRApy: COnstraints-Ba sed Reconstruction and Analysis for Python. BMC Systems Biology, 2013. 7(1): p. 74.

44. Ana conda-Software-Distribution. Ana conda. 2016, v 4.9.2. Ava ilable from: https://anaconda.com.

45. Rossum, G.v. and Python-Software-Foundation. Python tutorial, Technical Report CS-R9526. 1995, v 1. Availa ble from: http:// www.python.org.

46. Thiele, I. and B.Ø. Palsson, A protocol for generating a high-quality genomescale metabolic reconstruction. Nature Protocols, 2010. 5(1): p. 93-121.

47. Norsigian, C.J ., et al., A workflow for generating multi-strain genome-scale metabolic models of prokaryotes. Nature Protocols, 2020. 15(1): p. 1-14.

48. Seif, Y., et al., G enome-sc ale metabolic rec onstructions of multiple Salmonella strains reveal serovar-specific metabolic traits. Nature Communications, 2018. 9(1): p. 3771.

49. Monk, J.M., et al., iM L1515, a knowledgebase that computes Escherichia coli traits. Nature Biotechnology, 2017. 35(10): p. 904-908.

50. Monk, J.M., et al., Genome-sc ale metabolic reconstructions of multiple Escherichia coli stra ins highlight strain-specific a daptations to nutritional environments. Proceedings of the National Academy of Sciences, 2013. 110(50): p. 20338.

51. Lieven, C., et al., MEMOTE for standardized genome-sc ale metabolic model testing. Nature Biotechnology, 2020. 38(3): p. 272-276.

52. Tonkin-Hill, G., et al. Producing polished prokaryotic pangenomes with the Pana roo pipeline. Genome biology, 2020. 21, 180 DOI: 10.1186/s13059-02002090-4.

53. Kozlov, A.M., et al., RAXM L-NG : a fast, scalable and user-friendly tool for maximum likelihood phylogenetic inference. Bio informatics, 2019. 35(21): $p$. 4453-4455.

54. R-Core-Team. R: A Language and Environment for Statistic al Computing. 2020, v. Ava ilable from: https://www.R-project.org/.

55. RStudio-Team. RStudio: Integrated Development for R. 2020, v. Availa ble from: http:// www.rstudio.com/.

56. Neuwirth, E. RC olorBrewer: ColorBrewer Palettes. 2014, v 1.1-2. Ava ila ble from: https://cran.r-project.org/web/packages/RColorBrewer/ index.html.

57. Wickham, H., ggplot2: Elegant Graphic sor Data Analysis. 2016, New York: Springer-Verlag.

58. Wickham, H., Reshaping Data with the reshape Package. J oumal of Sta tistical Softwa re, 2007. 21(12).

59. Yu, G. a plot: Decorate a 'ggplot' with Associated Information. 2020, v 0.0.6. Ava ila ble from: https://CRAN.R-project.org/pa ckage=aplot.

60. Campitelli, E. ggnewsc ale: Multiple Fill and Colour Scales in 'ggplot2'. 2021, v 0.4.5. Ava ilable from: https://CRAN.R-project.org/pa ckage=ggnewscale.

61. Csardi, G. and T. Nepusz, The Igraph Software Package for Complex Network Research. Interj oumal, 2005. Complex Systems: p. 1695. 
62. Pedersen, T.L. Package 'ggraph'. 2021, v 2.0.5. Available from: https://CRAN.R-project.org/package=ggraph.

63. Pedersen, T.L. Package 'ggforce'. 2021, v 0.3.3. Available from: https://CRAN.R-project.org/package=ggforce.

64. Yu, G., et al., ggtree: an rpackage for visualization and annotation of phylogenetic trees with their covariatesand otherassociated data. Methods in Ecology and Evolution, 2017. 8(1): p. 28-36.

65. Kolde, R. pheatmap: Pretty Heatma ps. 2019, v 1.0.12. Available from: https://CRAN.R-project.org/package=phea tmap.

66. Auguie, B. and A. Antonov. Package 'gridExtra'. 2017, v 2.3. Available from: https://cran.r-project.org/web/packages/gridExtra/index.html.

67. Tidemann, C.R. and J.E. Nelson, Long-distance movements of the greyheaded flying fox (Pteropus poliocephalus). J oumal of Zoology, 2004. 263(2): p. 141-146.

68. Roberts, B.J ., et al., Long-Distance and Frequent Movements of the Flying-Fox Pteropus poliocephalus: Implic ations for Mana gement. PLOS ONE, 2012. 7(8): p. e42532.

69. Gomie, C.L., et al., Antimic robial-Resistant Klebsiella pneumoniae Ca miage and Infection in Specialized Geriatric Care Wards Linked to Acquisition in the Refeming Hospital. Clinical infectious disea ses: a n offic ial public ation of the Infec tious Disea ses Soc iety of Americ a, 2018. 67(2): p. 161-170.

70. Epstein, J.H., et al., Nipah vinus dyna mic sin bats and implications for spillover to humans. Proceedings of the National Aca demy of Sciences, 2020. 117(46): p. 29190-29201.

71. Welbergen, J .A., et al., Extreme mobility of the world's largest flying ma mmals creates key challenges for mana gement and conservation. BMC Biology, 2020. 18(1): p. 101.

72. Olson, M.E., et al., Sialic Acid Catabolism in Staphylococcus aureus. J oumal of Bacteriology, 2013. 195(8): p. 1779.

73. Manco, S., et al., Pneumococcal neuraminidases A and B both have essential roles during infection of the respiratory tract and sepsis. Infection and immunity, 2006. 74(7): p. 4014-4020.

74. Almagro-Moreno, S. and E.F. Boyd, Sialic Acid Catabolism Confers a Competitive Advantage to Pathogenic Vibrio cholerae in the Mouse Intestine. Infection and Immunity, 2009. 77(9): p. 3807-3816.

75. Chang, D.-E., et al., Carbon nutrition of Escherichia coli in the mouse intestine. Proc eedings of the National Aca demy of Sciences of the United States of America, 2004. 101(19): p. 7427.

76. Wyres, K.L., et al., Distinct evolutionary dyna mic s of horizontal gene transfer in drug resistant and virulent c lones of Klebsiella pneumoniae. PLOS G enetics, 2019. 15(4): p. e1008114.

77. Kanehisa, M. and S. Goto, KEG G: Kyoto Encyclopedia of Genes and Genomes. Nucleic AcidsResearch, 2000. 28(1): p. 27-30.

\section{Figure legends}

Figure 1: Sampled flying fox colonies a cross Australia by McDougall et al [1]. Coloured circles represent grey-headed flying fox colonies and the number within represents the number of Klebsiella pneumoniae species complex (KpSC) isolates obtained. KpSC were obtained from all colonies except 
for Centennial Park. Map of Australia generated by Lokal_Profil from https://upload.wikimedia.org/wikipedia/commons/b/bf/Australia map\%2C States.svg, used as per the Creative Commons Attribution-Share Alike 2.5 Generic lic ense.

Figure 2: Network graph showing pairwise single nucleotide variant (SNV) distances between isolates of the same sequence type. Isolates are shown as nodes, grouped by sequence type (ST) with the shape representing the species. The SNV distances are shown as lines between nodes. Isolates a re considered part of a transmission cluster if they have $\leq 25$ pairwise SNVs, represented by a solid line. Dashed lines show 26 - 100 painwise SNVs. Isolates are coloured based on their Grey-headed flying fox colony isolation source as indicated in the figure. The presence of the pFF1003-like plasmid is also indic ated.

Figure 3: MASH distance tree of plasmids found in the long read flying fox KpSC genomes. Tree was inferred from MASH distances using the FastME algorithm. Tip colour indicates grey-headed flying fox colony, while colour of plasmid label denotes the species as per legend in figure. The corresponding heatmap shows presence of antimicrobial resistance genes in pink, along with identity and coverage to the closest KpSC plasmid isolated from a human source, identified by Genbank's BLASTn. Accession numbers can be found in Table $\mathbf{S 4}$.

Figure 4: Comparison and distribution of ST4938 strains and associated plasmid FF1003 1. A Mid-point rooted core SNV chromosomal maximum-likelihood phylogeny of seven $K$. africana ST4938 isolates. Colony is indicted by tip colour. The heatmap shows plasmid SNV counts compared to the pFF1003_1 and pFF1003_2 completed reference plasmids. B Unrooted core chromosomal maximum likelihood daylight phylogeny of all flying-fox derived isolates included in the study with dots showing presence of pFF1003-like plasmids. Branch lengths were left unscaled to fit figure proportions. C Plasmid comparisons between pINF116 1 caried by $K$. pneumoniae INF116, isolated from a human source and flying fox plasmids pFF1003__1, pFF1010_1 and pFF1023_1. The orange a rrows indicate genes, blue arrows ind ic a te transposons and purple arrows indic ate metabolic genes such as the sialic acid catabolism nan locus. The grey bars between mapsindic ate nucleotide identity as per legend in the figure.

Figure 5: Mid-point rooted core genome maximum likelihood phylogeny of $72 \mathrm{KpSC}$ isolates and variable metabolic substrate use. Species are shaded on the phylogeny branches as per legend in the figure; $K a$ refers to $K$. africana, $K p$ refers to $K$. pneumoniae, Kqq refers to $K$. quasipneumoniae subsp. quasipneumoniae, $K$ qs refers to $K$. quasipneumoniae subsp. similipneumoniae, $K q v$ refers to $K$. quasivariicola, $K v t$ refers to $K$. variicola subsp. tropica, $K v v$ refers to $K$. variicola subsp. variicola. Broad ecological group/ isolation source is shown by tip colour. Flying fox isolates shown as light brown strips. Bootstrap values are shown in Figure S1. Variable predicted growth substrate utilisation is shown in the heatmap, coloured by substrate type as indic ated. No variable sulphur usage was predicted. 
bioRxiv preprint doi: https://doi.org/10.1101/2021.10.25.465810; this version posted October 26, 2021. The copyright holder for this preprint (which was not certified by peer review) is the author/funder, who has granted bioRxiv a license to display the preprint in perpetuity. It is made available under aCC-BY 4.0 International license. 


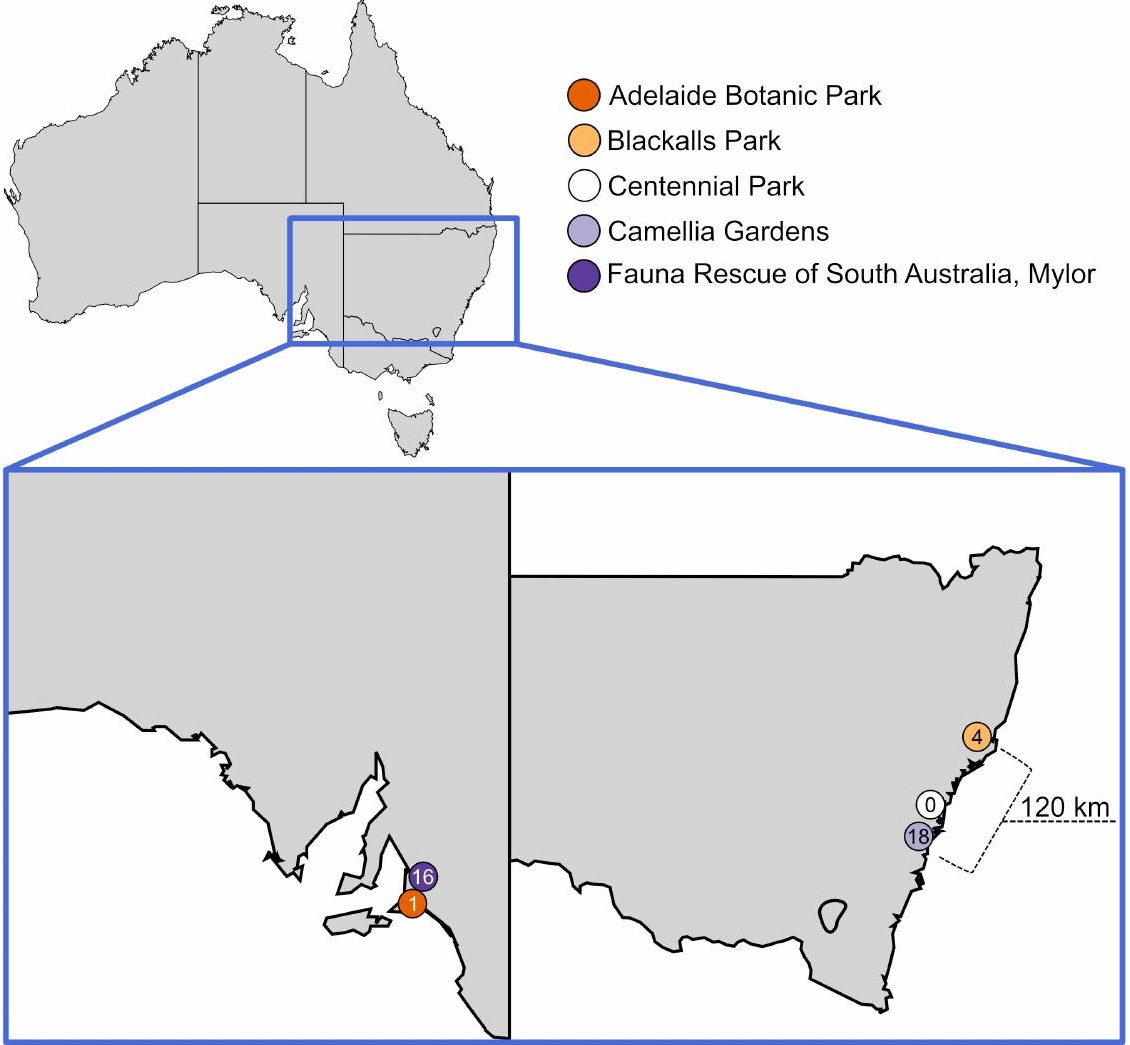




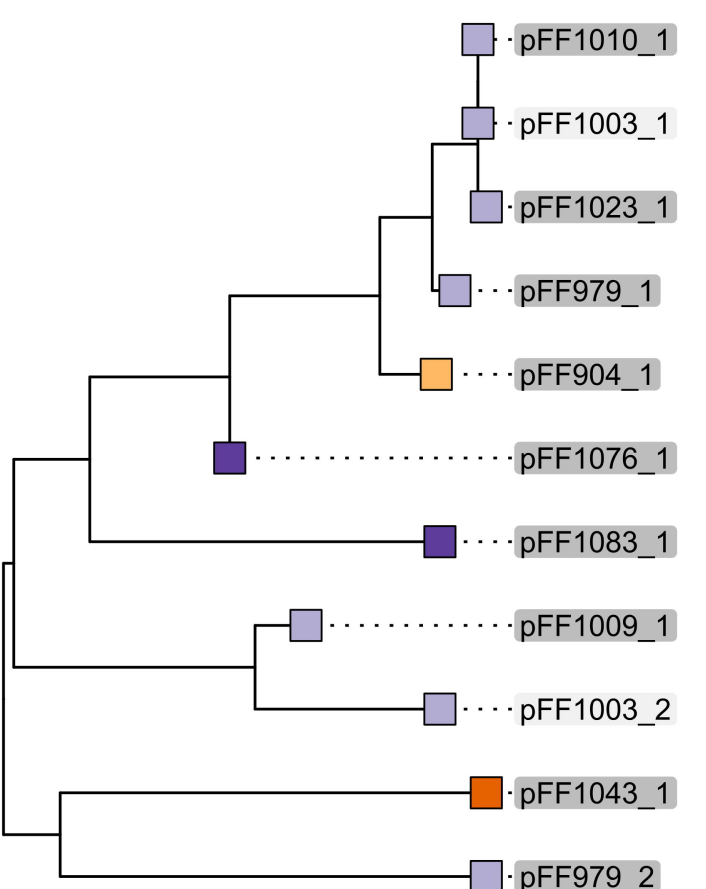

Non-mobilisable

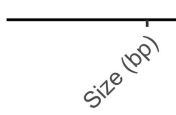

\section{Species}
a K. africana
a K. pneumoniae

Flying fox colony
$\square$ Adelaide
$\square$ Blackalls Park
$\square$ Camellia Gardens
Mylor

Antimicrobial resistance

0
1

Identity and coverage to closest human KpSC plasmids (\%)

$$
40
$$

60

80 

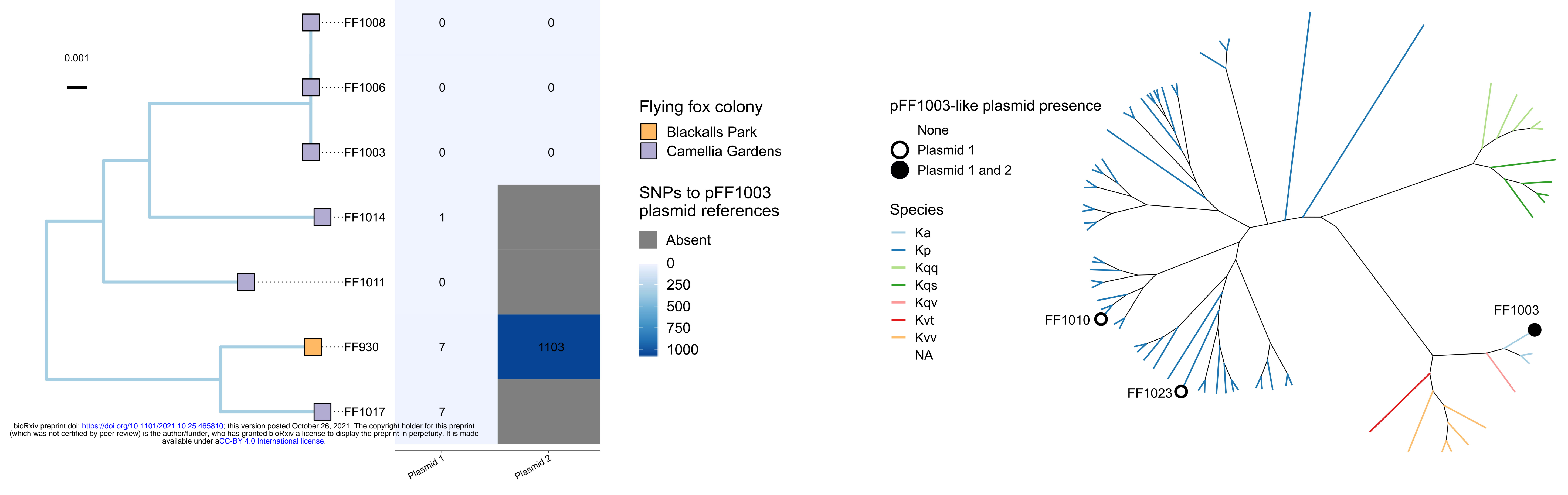

SNPs to pFF1003

plasmid references

Absent

0

250

750

1000

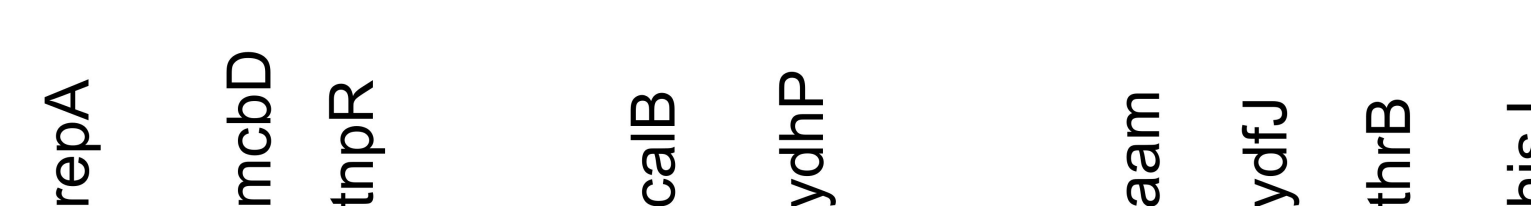

$\stackrel{m}{\text { है } \bar{~}}$

$\stackrel{\infty}{\infty} \frac{0}{\stackrel{0}{0}}$

O $\quad \begin{gathered}0 \\ 0\end{gathered}$

菏

pINF116_1

CP031793.1
K. pneumoniae

K. pneumonia
ST36

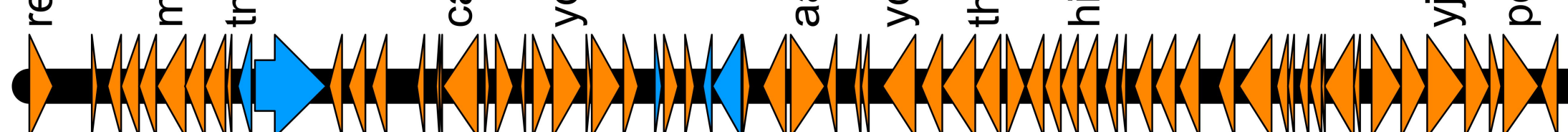

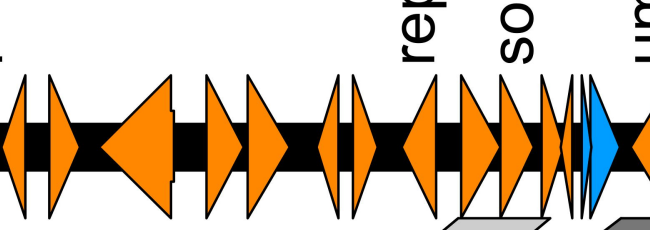

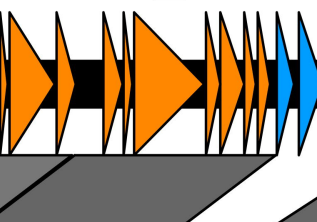

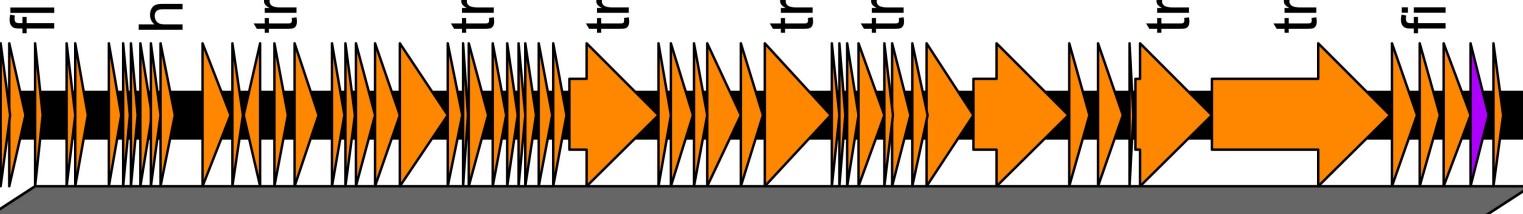

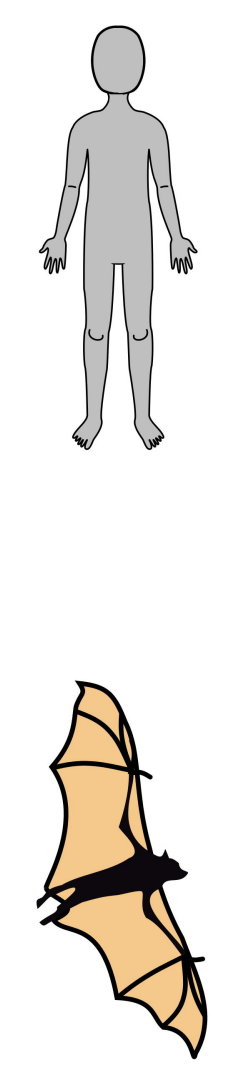

PFF1003 1 1

K. africana
ST 4938

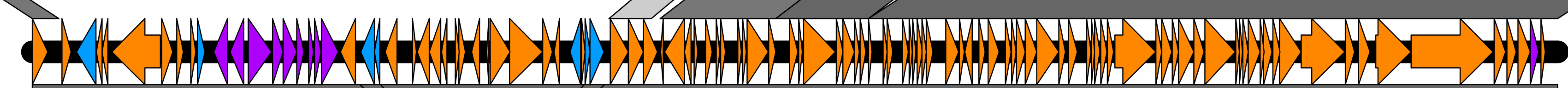

PFF1010_1

pFF1023_1

CP077818.1

K. pneumoniae
ST5037

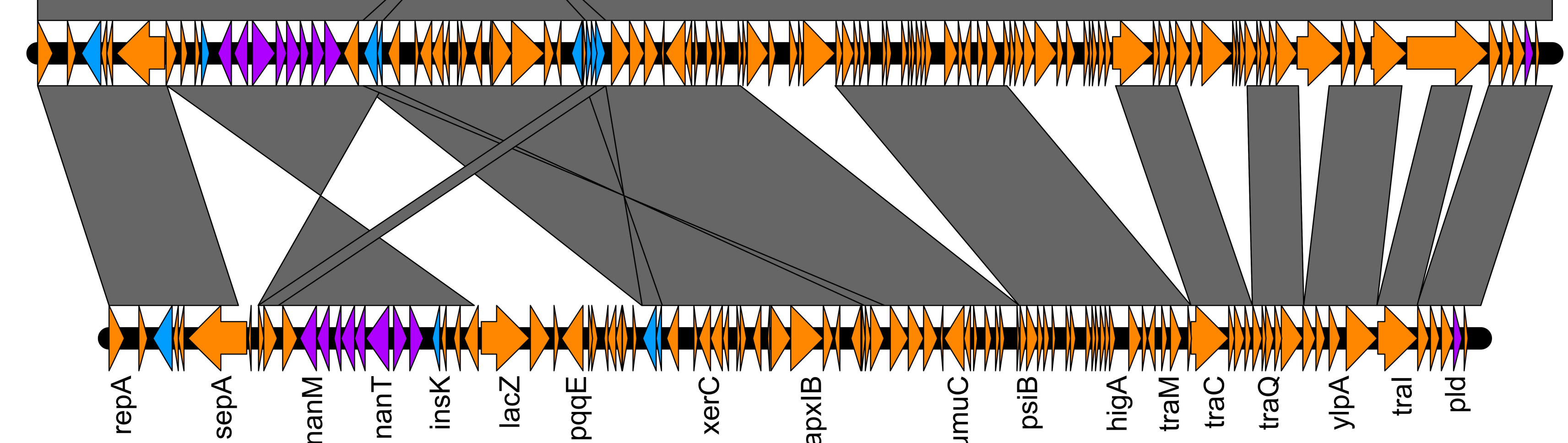


